\title{
Combined suppressive drug treatment in severe refractory rheumatoid disease: an analysis of the relative effects of parenteral methylprednisolone, cyclophosphamide, and sodium aurothiomalate
}

\author{
M T WALTERS AND M I D CAWLEY \\ From the Rheumatology Unit, Southampton General Hospital, Southampton
}

SUMMARY A trial was designed to assess the effects of intramuscular sodium aurothiomalate ot intravenous cyclophosphamide, or both, in combination with intravenous 'pulse' methylpredniso lone in severe intractable rheumatoid arthritis. Thirteen patients with severe, active rheumatoig arthritis, unresponsive to conventional therapeutic regimens showed improvement in synovitis after receiving a single intravenous bolus of methylprednisolone $(15 \mathrm{mg} / \mathrm{kg})$. Early mornin stiffness and Ritchie articular index remained improved over pretreatment values after 12 weeksథ

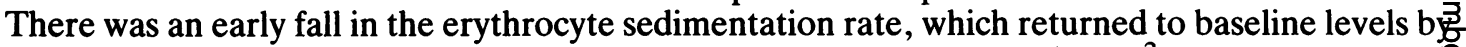
four weeks. A concomitant intravenous pulse of cyclophosphamide $\left(1 \mathrm{~g} / \mathrm{m}^{2}\right.$ body surface area果 given to eight patients did not confer any additional benefit. Six patients received sodignemo aurothiomalate, up to $100 \mathrm{mg}$ intramuscularly a week, and in these patients the ed $19^{\infty}$ improvement in synovitis induced by methylprednisolone was maintained. Thus between 12 啇要 24 weeks the Ritchie articular index, visual analogue pain score, erythrocyte sedimentation rates haemoglobin, and immunoglobin $\mathrm{G}$ were significantly better in the patients treated with gold an $\Phi$ methylprednisolone than in those treated with methylprednisolone alone, irrespective of whetheop they had received cyclophosphamide. Methylprednisolone pulse therapy given at the start of gol\$ treatment results in early improvement in synovitis, maintained until the usual delay in achievin $\overrightarrow{\mathcal{E}}$ a therapeutic effect from gold has elapsed.

Key words: rheumatoid arthritis, pulse therapy, synacthen test

Effective suppression of severe rheumatoid disease resistant to conventional treatment presents a difficult problem. By analogy with therapeutic regimens used in malignant disease, especially of the lymphoreticular system, with which rheumatoid disease has some features in common, ${ }^{1}$ it seemed possible that combinations of drugs in biologically effective doses might allow sufficient disease suppression to be achieved without unacceptable toxicity. Intermittent intravenous (IV) boluses or 'pulses' of methylprednisolone (MP) have been used for many years to suppress immunologically mediated disease. Renal allograft rejection can be prevented ${ }^{2}$ and idiopathic glomerulonephritis ${ }^{34}$ or the glomeru-

Accepted for publication 12 April 1988.

Correspondence to Dr M T Walters, Rheumatology Unit, Southampton General Hospital, Shirley, Southampton SO9 4XY. lonephritis associated with systemic lupus eryํㅗㅇ thematosus ${ }^{5}$ have responded well to this form of treatment, which has been shown to be superior toे high dose oral steroids. This approach has been extended to other autoimmune conditions, notably rheumatoid arthritis (RA), where it has been show that IV pulses of MP produce relief of symptoms and improvement in laboratory tests lasting for up tor three months. ${ }^{67}$ Oral cyclophosphamide has been shown to be of benefit in the treatment of active RA over an eight month period. ${ }^{8}$ When given for RA vasculitis as an intermittent IV bolus combined with MP, the side effects of cyclophosphamide are reduced without loss of therapeutic effect. ${ }^{9}$ Sodiun aurothiomalate is accepted by many as the standard against which other forms of suppressive treatment for RA should be compared, but it may need to b\$ given for several months before noticeable 
symptomatic improvement occurs. Once induced, remission may be prolonged for months. ${ }^{10}$

We suggest that MP given alone or combined with cyclophosphamide may induce a clinical remission in severe, active RA, which could then be maintained in the medium to long term by gold treatment.

\section{Patients and methods}

Patients entering the study fulfilled the American Rheumatism Association criteria for classical or definite RA, and all had severe, active, seropositive and erosive disease. Active disease was defined by the presence of at least three of the following five criteria: (a) erythrocyte sedimentation rate (ESR, Westergren) $>50 \mathrm{~mm} / 1 \mathrm{st} \mathrm{h}$; (b) Ritchie articular index (RAI) $>25$ (maximum score obtainable on our modified version was 78 ); (c) early morning stiffness (EMS) $>30$ minutes; $(d)$ visual analogue pain score on $100 \mathrm{~mm}$ horizontal scale (VAPS) $>30$; (e) haemoglobin $<110 \mathrm{~g} / \mathrm{l}$. Twelve of the 13 patients analysed fulfilled at least four of these criteria. All patients had failed to respond to an adequate trial of at least one other second line drug in conventional doses, including gold (at least six months' treatment, including six weeks receiving $50 \mathrm{mg}$ intramuscularly (IM) weekly), D-penicillamine (at least six months' treatment, including six weeks receiving $500 \mathrm{mg}$ daily), azathioprine (at least three months' treatment, including six weeks receiving $150 \mathrm{mg}$ daily), and hydroxychloroquine (at least three months' treatment receiving $400 \mathrm{mg}$ daily).

Patients who had received systemic corticosteroids during the previous two years and those with dyspepsia or a history of intolerance to corticosteroids or gold salts were excluded from the study.

No drugs other than non-steroidal antiinflammatory compounds, simple analgesics, or drugs for non-rheumatological conditions were allowed in the month before entry into the study.

Patients were admitted to hospital for two weeks. On admission a full clinical examination was performed and the following recorded: body weight, duration of EMS, VAPS, grip strength (GS), RAI, and range of joint movement, in particular shoulder abduction and knee flexion. Laboratory investigations included full blood count, ESR, $C$ reactive protein (CRP), and immunoglobulins $G, A$, and $M$. A short synacthen test was performed on admission and after 24 weeks.

Patients were randomly allocated to one of four groups and received treatment as follows: group A-methylprednisolone sodium succinate $15 \mathrm{mg} / \mathrm{kg}$ body weight, given as a single IV bolus in $100 \mathrm{ml}$ of isotonic saline over 30 minutes. Group B-As for group A together with cyclophosphamide $1 \mathrm{~g} / \mathrm{m}^{2}$ body surface area as an IV bolus immediately before the MP. Group C-As for group A with IM sodium aurothiomalate as follows: day 1-test dose $10 \mathrm{mg}$, day 5-50 mg, day 9-100 mg, day $13-100 \mathrm{mg}$. Group D-As for group C with cyclophosphamide 1 $\mathrm{g} / \mathrm{m}^{2}$ as an IV bolus as in group $B$.

Patients were rested in bed for the first week and then gradually mobilised during the second week.

After discharge from hospital groups $\mathrm{C}$ and $\mathrm{D}$ continued to receive gold injections $50 \mathrm{mg}$ weekly. Clinical and laboratory assessments were repeated at 2, 4, 8, 12, 16, 20, and 24 weeks. After 12 weeks all patients in all four groups were offered a second pulse of MP, and those requesting this were readmitted to hospital for a further two week period. Other treatment continued unchanged.

\section{STATISTICAL ANALYSIS}

The Mann-Whitney U test for non-parametric data was used to analyse the unpaired data as follows: (1) The effect of a single pulse of MP and two weeks hospitalisation on the clinical and laboratory parameters of active RA. The combined baseline values for all groups were compared with the combined values for all groups at $2,4,8$, and 12 weeks. (2) The effect of the addition of an IV pulse of cyclophosphamide to the MP. The values for groups $A$ and $C$ (no cyclophosphamide) were combined and compared with the combined values for groups B and D (cyclophosphamide treated), at 2, 4, 8, and 12 weeks. (3) The effect of adding gold to either MP or MP plus cyclophosphamide pulse therapy. The combined values for groups A and B (no gold) were compared with the combined values for groups $\mathrm{C}$ and $\mathrm{D}$ (gold treated) at $4,8,12,16,20$, and 24 weeks.

\section{Results}

Seventeen patients were entered and 13 patients completed the study. Table 1 shows the demographic details.

Of the initial 17 patients, two group A patients withdrew because they felt the treatment was ineffective, one after two weeks and the other after

Table 1 Demographic data of patients in the study. Mean values (range) are given

\begin{tabular}{llll}
\hline Group & Age (years) & $\begin{array}{l}\text { Duration of } \\
\text { disease (years) }\end{array}$ \\
\hline A (4F, 1M) & 56 & $(44-69)$ & $12 \cdot 6(2-27)$ \\
B (4F) & $55 \cdot 5(42-63)$ & $12 \cdot 3(6-18)$ \\
C (3F, 1M) & 61 & $(54-74)$ & $12 \cdot 7(5-17)$ \\
D (2F, 2M) & 61 & $(52-72)$ & $12 \cdot 3(3-24)$ \\
\hline
\end{tabular}


12 weeks; one group $\mathrm{C}$ patient entered as a penicillamine 'failure' developed an acute exacerbation of synovitis six weeks after stopping the drug and subsequently restarted penicillamine; one group D patient was withdrawn because of the development of the nephrotic syndrome after $310 \mathrm{mg}$ of gold. A renal biopsy showed minimal change glomerulonephritis and the nephrosis has since slowly resolved. Side effects attributable to the MP within the 24 hour period after the infusion were frequent but usually minor and included (occurrences in parentheses) facial flushing (four), feelings of faintness or clamminess (three), headaches (one), palpitations (one), bitter taste in mouth (three), depression (one), and an exacerbation of synovitis (two). One patient with a history of two previous myocardial infarctions and stable angina had a small anterolateral myocardial infarction within 36 hours of the infusion. Five of the eight patients treated with cyclophosphamide had nausea or vomiting, or both, within 12 hours of the infusion. In six of these eight patients there was a mild neutropenia after tw®. weeks (mean neutrophil count $1.38 \times 10^{9} / 1$, range? $\left.0.92-1.95 \times 10^{9} / 1\right)$, which reverted to normal by fou $\overrightarrow{\vec{s}}$ weeks.

EFFECT OF A SINGLE IV PULSE OF MP We compared the baseline entry values of bod weight, duration of EMS, VAPS, GS, RAI, range of shoulder abduction and knee flexion, haemoglobino, ESR, CRP, IgG, IgA, and IgM in all groups anக் found no significant differences.

When the combined baseline values for all groups. were compared with the combined values for alo groups at $2,4,8$, and 12 weeks there were significan $\bar{p}$ improvements in EMS, VAPS, and RAI within 12 weeks after a single pulse of MP (Figs 1a-c). The mean value for the ESR fell two weeks after the pulse was given from $81 \mathrm{~mm} / 1 \mathrm{st} h$ to $63 \mathrm{~mm} / 1 \mathrm{st} h \mathrm{~h}_{\perp}^{N}$ but this was not statistically significant $(p=0.06)$. Alg short synacthen tests were normal on entry into the study and again after 24 weeks.

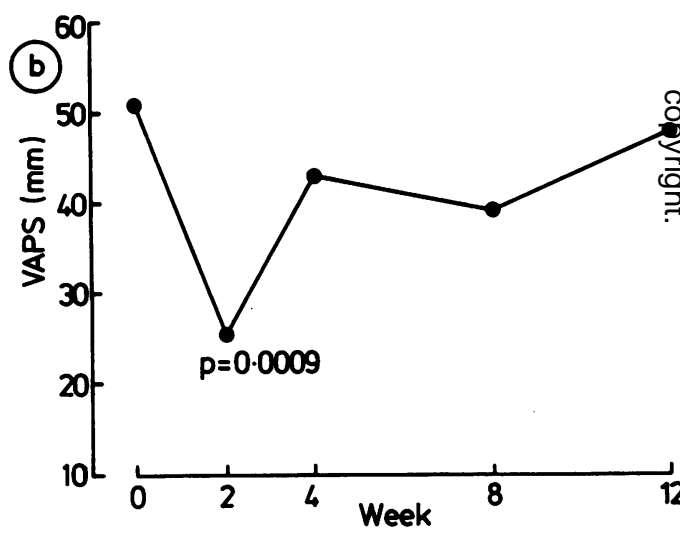

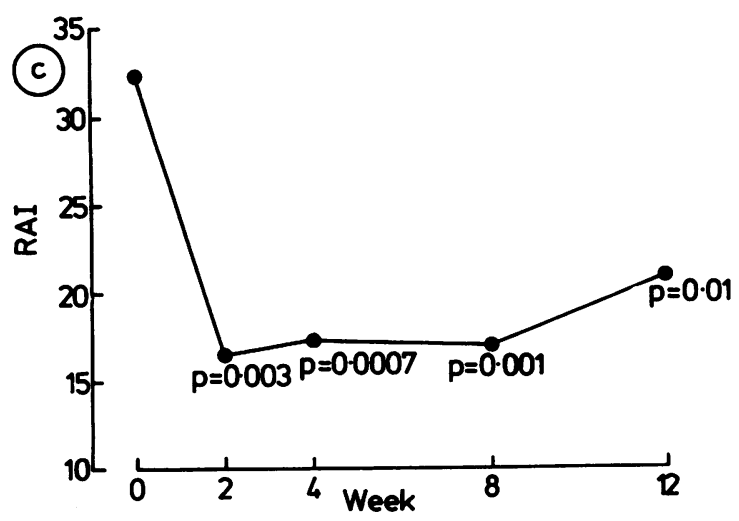

Fig. 1 Effect of intravenous methylprednisolone $(15 \mathrm{mg} / \mathrm{kg})$ on measurements of disease activity in patients with rheumatoid arthritis in groups $A, B, C$, and $D$ combined $(n=17)$. (a) Early morning stiffness (EMS); (b) visual analogue pain score (VAPS); (c) Ritchie articular index (RAI). 
EFFECT OF A SINGLE IV PULSE OF CYCLOPHOS PHAMIDE

When the values for groups $\mathrm{A}$ and $\mathrm{C}$ (no cyclophosphamide) were combined and compared with the combined values for group B and D (cyclophosphamide treated) at $2,4,8$, and 12 weeks there were no statistically significant differences, suggesting that a single pulse of cyclophosphamide did not confer
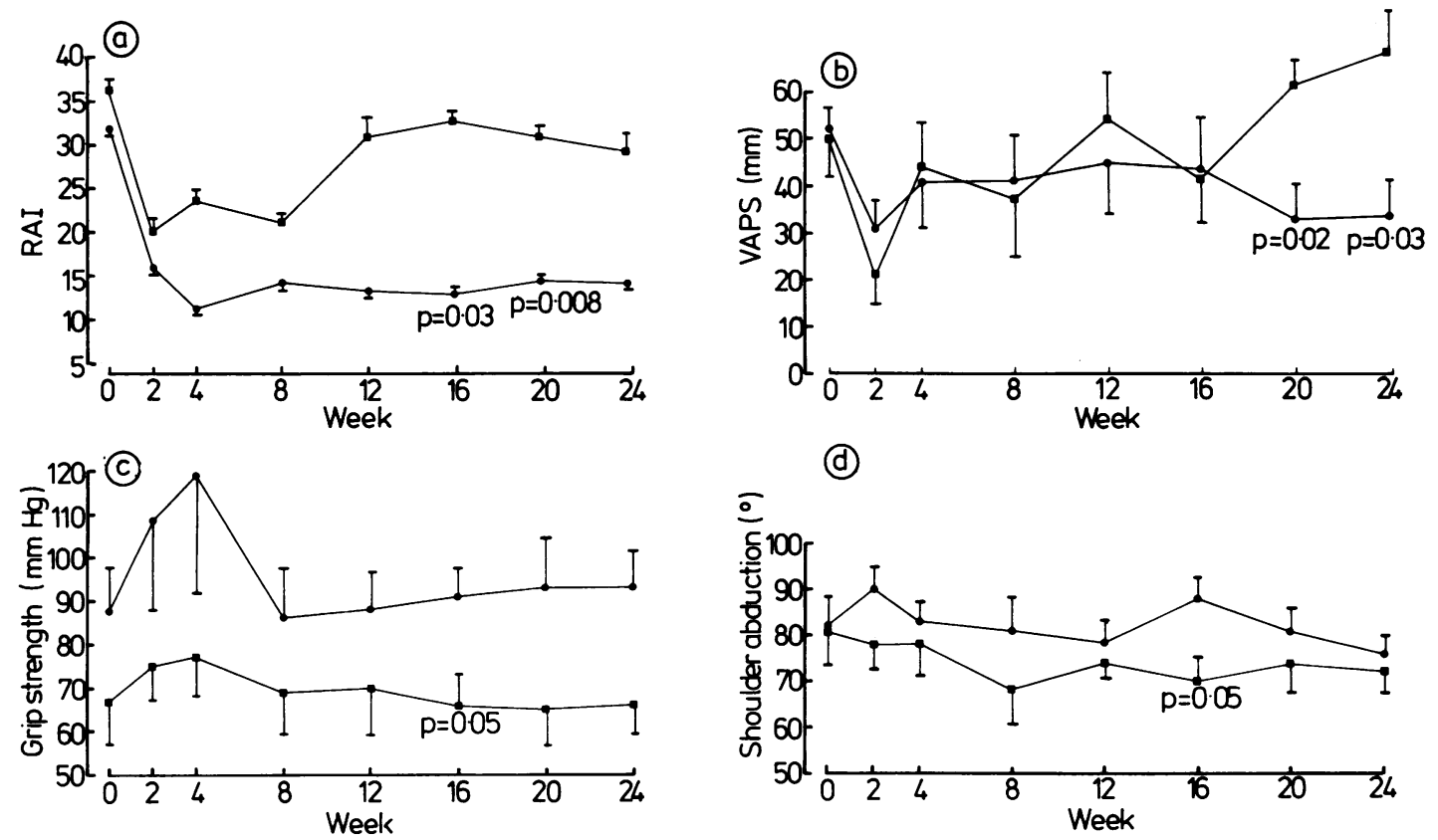

(d)
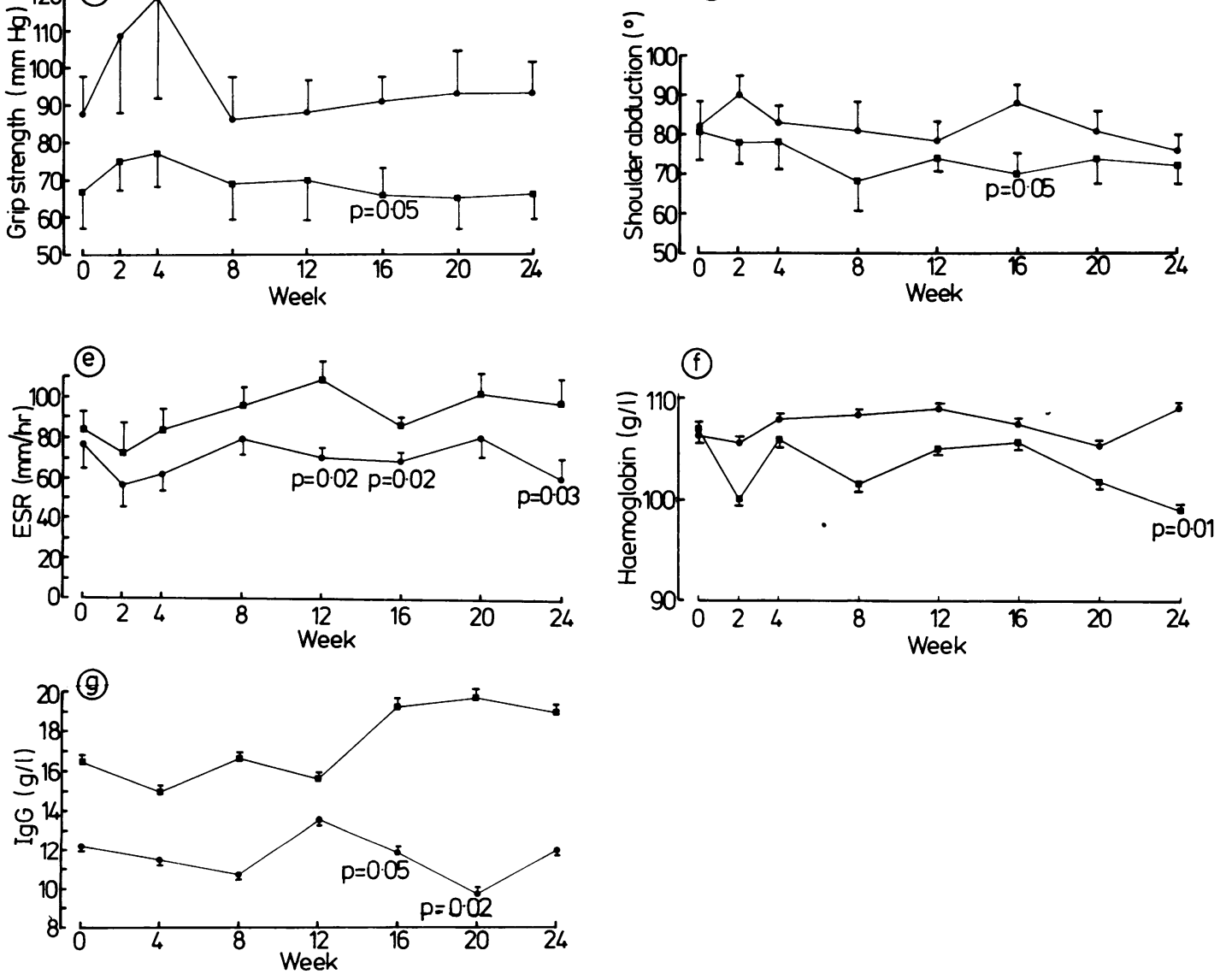

Fig. 2 Comparison of measurements of disease activity in groups $A$ and $B$ (no gold) $\square$ and groups $C$ and $D$ (gold treated) - during 24 weeks after intravenous methylprednisolone (15 mg/kg). (a) Ritchie articular index (RAI); (b) visual analogue pain score (VAPS); (c) grip strength; (d) shoulder abduction; (e) erythrocyte sedimentation rate (ESR); $(f)$ haemoglobin; $(g)$ immunoglobulin $G$. Error bars indicate the standard error of the mean. 
additional benefit over a single pulse of MP in decreasing disease activity, at least over a 12 week period.

EFFECT OF THE ADDITION OF GOLD

As the therapeutic benefit of gold salts in RA usually takes several weeks or months to become apparent we compared the combined mean values for each parameter in groups $A$ and $B$ (no gold) and groups $C$ and $D$ (gold treated) at 2, 4, 8, 12, 16, 20 and 24 weeks after the pulse of MP with or without cyclophosphamide and initiation of gold treatment. Two group A and one group B patient received further pulses of MP at 12 weeks, whereas no patient in groups $\mathrm{C}$ or $\mathrm{D}$ elected to have this. Despite this, groups C and D (gold treated) showed better overall improvement.

When the clinical indices of disease activity were examined, there was a difference between the combined groups in favour of gold treatment in terms of RAI as early as week 4 (Fig. 2a), and this was statistically significant from week $16(p=0.03)$. At weeks 20 and 24 the group $C / D$ patients complained of significantly less overall pain in their joints (VAPS) $(\mathrm{p}=0.02$ and 0.03 respectively) (Fig. 2b). At week 16 GS was significantly higher in the gold treated group (Fig. 2c), and the range of glenohumeral joint movement had increased $(p=0.05$; Fig. 2d). There were no significant changes over the 24 week observation period in the following parameters: knee flexion (range group A/B $126-138^{\circ}$, range group C/D $116-125^{\circ}$ ), body weight (range group A/B $58 \cdot 0-60 \cdot 7 \mathrm{~kg}$, range group C/D $56 \cdot 8-64.7 \mathrm{~kg}$ ), and EMS (range group A/B $21 \cdot 7-56 \cdot 0$ minutes, range group $C / D$ 21.4-47.1 minutes).

When the laboratory indices of disease activity were examined, from week 12 there was a significant difference in the ESR between the combined groups in favour of the gold treated group, which was maintained until the end of the study (Fig. 2e). The mean haemoglobin concentration was significantly higher in the group C/D patients at 24 weeks (109v $99 \mathrm{~g} / \mathrm{l}, \mathrm{p}=0.01$ ) (Fig. 2f). Although the absolute value of serum IgG did not change in the group C/D patients, the concentration tended to rise in the group $A / B$ patients, so that at weeks 16 and 20 the differences were significant $(p=0.05$ and $p=0.02$ respectively; Fig. 2g). There were no significant differences between the groups in serum IgA (range group $A / B 2 \cdot 2-4 \cdot 7 \mathrm{~g} / 1$, range group $C / D 3.0-4 \cdot 5 \mathrm{~g} / \mathrm{l})$, nor in serum IgM (range group A/B $1.8-2.8 \mathrm{~g} / \mathrm{l}$, range group $C / D$ $0 \cdot 6-1 \cdot 1 \mathrm{~g} / \mathrm{l})$. Similarly, there were no significant differences between the groups in serum CRP (range group A/B 0.038-0.1 g/l, range group $C / D \quad 0 \cdot 057-0 \cdot 123 \mathrm{~g} / \mathrm{l})$.

\section{Discussion}

This study confirms the findings of others that pulse MP is an effective short term treatment for mosf patients with active RA. ${ }^{67}$ Moreover pulse MPE therapy given at the start of chrysotherapy mayen eliminate the 'lag' period before the improvement in disease due to gold becomes apparent. There have been two recent comparable studies. Neumann et a fis observed a beneficial clinical effect when pulsed MPS was given at the start of treatment with penicilla mine or sulphasalazine, ${ }^{11}$ and Hansen et al found $\vec{\omega}$ clinical benefit and early falls in the ESR, VAPS and RAI after pulsed MP given before gold, penicillamine, or azathioprine. ${ }^{12}$ In both these studies, however, three IV pulses of MP were given:We used only a single IV pulse of MP at the start of treatment, which in most patients produced sustained improvement. In some of the patients nop receiving gold the effect was short lived and a second dose of MP was given after 12 weeks if the patient elected to receive this.

situation is not clear. There is some evidence tha MP given in this way may be immunosuppressive a after infusion there is a profound peripheral blogd. lymphopenia after four hours followed by a ret of lymphocytes to normal levels by 24 hours. 131.100 Alternatively, MP may merely induce a sufficientity prolonged anti-inflammatory effect, which lasts untif the suppressive effect of gold becomes manifest

Side effects attributable to MP were usuall: minor, but one patient had palpitations and anothe a small myocardial infarction shortly after the pulset of MP. Neumann et al also mention that one of their patients was withdrawn from their study owing to a myocardial infarction. ${ }^{11}$ They felt that the pulse therapy was not responsible, but no details wers given. We would advise caution in the use of MP foo the treatment of patients with a history of ischaemis heart disease in view of the positive inotropic effect of the drug on the myocardium. We performed shore synacthen tests on all patients to detect possible suppression of the hypothalamic-pituitary-adren $\$$ axis but found no evidence of this.

Oral cyclophosphamide has been of benefit i severe intractable RA, ${ }^{15-17}$ but the possibility of inducing serious long term side effects, such as haemorrhagic cystitis, ${ }^{18}$ carcinoma of the bladde and leukaemia or lymphoma, ${ }^{19} 20$ has cause ${ }^{2}$ concern. There are few published data on the use intermittent IV pulses of cyclophosphamide io active RA, although when combined with MP it has improved rheumatoid vasculitis, with a low inc f $^{\circ}$ dence of side effects. ${ }^{9}$ We hoped that the combinat tion of cyclophosphamide, with or without gold, and MP might have an additional synergistic effect of 
rheumatoid synovitis. The addition of a single pulse of cyclophosphamide to the MP, however, conferred no more benefit than a single pulse of MP, but caused no serious side effects. Minor side effects such as nausea and vomiting occurred in five of eight patients. The degree of neutropenia was acceptable. Continuous high dose oral administration of cyclophosphamide is often limited by toxicity. It is possible that more frequent, intermittent IV boluses may be more beneficial than the single IV bolus we used. One complication of gold treatment occurred in the patient who developed nephrosis after 310 $\mathrm{mg}$, which gradually resolved after withdrawal of the gold.

Our study suggests that in severe, active RA, when treatment with gold is contemplated, a single IV pulse of MP given at the onset of treatment is usually effective in inducing an early remission of synovitis before the effect of the second line agent. Neumann et al used three alternate day pulses of IV MP over five days, ${ }^{11}$ and Hansen et al used three IV pulses of MP on consecutive days. ${ }^{12}$ We agree with Hansen et $\mathrm{l}^{12}$ that this may be no more beneficial in most patients than a single IV bolus and suggest that as there are potential risks from this form of treatment that a single pulse of MP should be tried in the first instance. Subsequently, it may be appropriate to repeat the pulse therapy after an interval, depending on the individual clinical response. In our experience the side effects of this form of treatment for severe RA are usually minor. We would, however, urge caution in patients with ischaemic heart disease.

The authors gratefully acknowledge financial support from the Southampton Rheumatic Disease Research Fund, technical assistance from the staff of the departments of chemical pathology, immunology, and occupational therapy at Southampton General Hospital, and the cooperation of the participating patients.

\section{References}

1 Bitter T. A new look at rheumatoid arthritis. In: Wright V, ed. Topical reviews in rheumatic disorders. Vol. 2. Bristol: Wright, 1982: 1-74.

2 Feduska N J, Turcotte J G, Gikas P W, Bacon G E, Penner J A. Reversal of renal allograft rejection with intravenous methylprednisolone 'pulse' therapy. J Surg Res 1972; 12: 208-15.
3 Oredugba O, Mazumdar D C, Meyer J S, Lubowitz H. Pulse methylprednisolone therapy in idiopathic, rapidly progressive glomerulonephritis. Ann Intern Med 1980; 92: 504-6.

4 Cole B R, Brocklebank J T, Kienstra R A, Kissane J M, Robson A M. 'Pulse' methylprednisolone therapy in the treatment of severe glomerulonephritis. J Pediatr 1976; 88: 307-14.

5 Cathcart E S, Scheinberg M A, Idelson B A, Couser W G. Beneficial effects of methylprednisolone 'pulse' therapy in diffuse proliferative lupus nephritis. Lancet 1976; i: 163-6.

6 Liebling M R, Lieb E, McLaughlin K, et al. Pulse methylprednisolone in rheumatoid arthritis. Ann Intern Med 1981; 94: 21-6.

7 Williams I A, Bayliss M E, Shipley M E. A double-blind placebo-controlled trial of methylprednisolone pulse therapy in active rheumatoid disease. Lancet 1982; ii: 237-40.

8 Cooperating clinics committee of the American Rheumatism Association. A controlled trial of cyclophosphamide in rheumatoid arthritis. N Engl J Med 1970; 283: 883-9.

9 Scott D G I, Bacon P A. Intravenous cyclophosphamide plus methylprednisolone in treatment of systemic rheumatoid vasculitis. Am J Med 1984; 76: 377-84.

10 Research subcommittee of the Empire Rheumatism Council. Gold therapy in rheumatoid arthritis. Final report of a multicentre controlled trial. Ann Rheum Dis 1961; 20: 315-34.

11 Neumann V, Hopkins R, Dixon J, Watkins A, Bird H, Wright $\mathrm{V}$. Combination therapy with pulsed methylprednisolone in rheumatoid arthritis. Ann Rheum Dis 1985; 44: 747-51.

12 Hansen T M, Dickmeiss E, Jans H, Hansen T I, IngemenNielsen M, Lorenzen I. Combination of methylprednisolone pulse therapy and remission inducing drugs in rheumatoid arthritis. Ann Rheum Dis 1987; 46: 290-5.

13 Webel M L, Ritts R E, Taswell H F, Donadio J V, Woods J E. Cellular immunity after intravenous administration of methylprednisolone. J Lab Clin Med 1974; 83: 383-92.

14 Fauci A S, Dale D C. The effect of in vivo hydrocortisone on subpopulations of human lymphocytes. J Clin Invest 1974; 53: 240-6.

15 Fosdick W M, Parsons J L, Hill D F. Long term cyclophosphamide therapy in rheumatoid arthritis. Arthritis Rheum 1968; 11: 151-61.

16 Horslev-Petersen K, Beyer J M, Helin P. Intermittent cyclophosphamide in refractory rheumatoid arthritis. $\mathrm{Br} \mathrm{Med} \mathrm{J}$ 1983; 287: 711-2.

17 Smyth C J, Bartholomew B A, Mills D M, Steigerwald J C, Strong S J, Recart S. Cyclophosphamide therapy for rheumatoid arthritis. Arch Intern Med 1975; 135: 789-93.

18 Marshall F F, Klinefelter H F. Late hemorrhagic cystitis following low-dose cyclophosphamide therapy. Urology 1979; 14: $573-5$.

19 Hazleman B. Incidence of neoplasms in patients with rheumatoid arthritis exposed to different treatment regimens. $A m ~ J$ Med 1985; 78: 39-43.

20 Decker J L. Azathioprine and cyclophosphamide as slow acting drugs for rheumatoid arthritis. Am J Med 1983; 75: 74-8. 
5 Shu K H, Lian J D, Yang Y F, et al. Glomerulonephritis in ankylosing spondylitis. Clin Nephrol 1986; 25: 169-74.

6 Mittal V K, Malhotra K K, Bhuyan U N, Malaviya A N. Kidney involvement in seronegative spondarthritides. Indian J Med Res 1983; 78: 670-5.

7 Tiebosch A T M G, Wolters J, Frederik P F M, et al. Epidemiology of idiopathic glomerular disease: a prospective study. Kidney Int 1987; 32: 112-6.

8 Simon P, Ang K S, Bavay P, Cloup C, Mignard J P, Ramee M P. Glomérulonéphrite à immunoglobulines A. Epidémiologie dans une population de 250,000 habitants. Presse Med 1984; 13: $257-60$.

\section{Alterations in appendicular skeletal mass in patients with rheumatoid arthritis, psoriatic arthritis, and osteoarthritis}

SIR, Cooper $e t$ al in their recent article on skeletal mass in rheumatoid arthritis, psoriatic arthritis, and osteoarthritis stated that lumbar bone mass is reduced in rheumatoid arthritis, irrespective of corticosteroid treatment. ${ }^{1}$

For this statement they quoted, without any personal experience, one published reference. ${ }^{2}$ In our opinion, however, this statement does not reflect the results of other reports concerning lumbar bone mass in rheumatoid arthritis. From the same author as the one referred to by Cooper $e$ al there is a previous study of early rheumatoid arthritis, in which no diminution of bone mass could be shown when patients with rheumatoid arthritis were compared with controls. ${ }^{3}$ In our own study we found a normal lumbar bone mass when female, postmenopausal patients with rheumatoid arthritis were compared with controls matched for sex, age, and menopausal state, irrespective of corticosteroid treatment.

Moreover, when discussing the appendicular skeleton as measured by single photon absorptiometry the authors refer to 'one single study', whereas there are many studies of peripheral bone mass at the radial site in rheumatoid arthritis, treated with corticosteroids or not. ${ }^{4-11}$ Some authors report a decreased peripheral bone mass in rheumatoid arthritis while others do not.

As the assessment of bone mass in arthritis is difficult owing to a variety of interfering factors, such as sex, age, menopausal state, disease activity and duration, local destruction, treatment (especially corticosteroid treatment), I feel that the discussion should have been expanded, with a better use of the available publications and a discussion of current controversies.

Arthritis and Metabolic Bone

Disease Research Unit,

A VERSTRAETEN

K U Leuven, U Z. Pellenberg,

B-3041 Pellenberg,

Belgium

\section{References}

1 Cooper C, Poll V, McLaren M, Daunt S O'N, Cawley M I D. Alterations in appendicular skeletal mass in patients with rheumatoid, psoriatic, and osteoarthropathy. Ann Rheum Dis 1988; 47: 481-4.

2 Sambrook P N, Eisman J A, Yeates M G, Pocock N A, Eberl S, Champion G D. Osteoporosis in rhcumatoid arthritis: safety of low dose corticosteroids. Ann Rheum Dis 1986; 45: 950-3.

3 Sambrook P N, Ansell B M, Foster S, Gumpel J M, Hesp R, Reeve J. Bone turnover in early rheumatoid arthritis. 2. Longitudinal bone density studies. Ann Rheum Dis 1985; 44: $580-4$.

4 Verstraeten A, Dequeker J. Vertebral and peripheral bone mineral content and fracture incidence in postmenopausal patients with rheumatoid arthritis: effect of low dose corticosteroids. Ann Rheum Dis 1986; 45: 852-7.

5 Christansen C, Rodbro P. Skeletal status in patients with rheumatoid arthritis. Acta Med Scand 1975; 198: 453-4.

6 D'Angelo A, Fabris A, Sartori L, et al. Mineral metabolism and bone mineral content in rheumatoid arthritis. Effect of corticosteroids. Clin Exp Rheumatol 1985; 3: 143-6.

7 Dequeker J, Wielandts L, Koentges D, Nijs J. The assessment of bone loss in rheumatoid arthritis. Acta Rhumatologica 1980; 4: 228-9.

8 Mueller M N. Effects of corticosteroids on bone mineral in rheumatoid arthritis and asthma. AJR 1976; 126: 1300.

9 Nagant de Deuxchaisnes C, De Vogelaer J P, Esselinckx W, et al. The effect of low dose glucocorticoids on bone mass in rheumatoid arthritis: a cross-sectional and longitudinal study using single photonabsorptiometry. In: Alvioli L, Gennari C, Imbimbo $\mathrm{H}$, eds. Glucocorticoid effects and their biological consequences. New York: Plenum Press, 1984: 209-39.

10 Als O S, Godfredsen A, Christiansen C. The effect of glucocorticosteroids on bone mass in rheumatoid arthritis patients. Influence of menopausal state. Arthritis Rheum 1985; 28: $369-75$.

11 Als O S, Christiansen C, Hellesen C. Prevalence of decreased bone mass in rheumatoid arthritis. Relation to antiinflammatory treatment. Clin Rheumatol 1984; 3: 201-8.

SIR, As Dr Verstraeten indicates studies of bone mass at different anatomical sites in rheumatoid arthritis have produced conflicting results, and we stated this in our report. It was not our purpose to write an exhaustive review of published work but to highlight some of the inconsistencies, which are, quite naturally, open to various interpretations. We reported our own measurements of appendicular bone mass in three different polyarthropathies and considered these to be of interest. Dr Verstraeten's comments on corticosteroid treated patients do not really apply in this context owing to the deliberate exclusion of such patients from our study.

Rheumatology Unit and

C COOPER

MRC Environmental Epidemiology Unit, M MCLAREN Southampton General Hospital, Southampton SO9 4XY

S O'N DAUNT M I D CAWLEY

Correction: Combined suppressive drug treatment in severe refractory rheumatoid disease: an analysis of the relative effects of parenteral methylprednisolone, cyclophosphamide, and sodium aurothiomalate. In the paper by Drs M T Walters and M I D Cawley (Ann Rheum Dis 1988; 47: 924-9) we regret that the first line of the second paragraph of the Discussion was omitted. The first sentence of this paragraph should have read 'The mode of action of both gold and MP in this situation is not clear'. 